\title{
Pseudo Weakly N-Projective Modules
}

\author{
Sushma Jat, Vivek Patel
}

\begin{abstract}
A module $M$ is said to be weakly projective iff it has a projective cover $\pi: P(M) \longrightarrow M$ and every mapping $P(M)$ into a finitely generated module can be factored through $M$ via an epimorphism. In particular, if $M$ and $N$ are two $R$-modules and assume $M$ has a projective cover $\pi: P \longrightarrow M$, We say that $M$ is pseudo weakly $N$-projective if for every map $\psi: P \longrightarrow N$ there exists an epimorphism $\sigma: P \longrightarrow M$ and $a$ homomorphism $g: M \longrightarrow N$ such that $\psi=g . \pi$. In this paper we generalize the basic properties of pseudo weakly projective modules.
\end{abstract}

Keywords: Pseudo projective module, pseudo weakly projective module, Projective cover.

\section{Definition:1}

An R-module $\mathrm{M}$ is said to be pseudo projective if for any given R-module A, epimorphisms $g: M \rightarrow A$ and $f: M \rightarrow A$ there exists a homomorphism $h: M \rightarrow M$ such that $f=g \cdot h$

\section{Definition :2}

We say that $\mathrm{M}$ is weakly M-Projective [Quasi Weakly Projective), If $\mathrm{M}$ has a projective cover $\pi: P(M) \rightarrow M$ and every homomorphism $\Psi: P(M) \rightarrow N$ can be factored through $\mathrm{M}$ via some epimorphism. Equivalently, a module $\mathrm{M}$ is weakly $\mathrm{M}$ projective if it has a projective cover $\pi: P(M) \rightarrow M$ and given any homomorphism $\Psi: P(M) \rightarrow N$ there exists $X \subseteq$ ker $\Psi$ such that $\frac{P(M)}{X} \cong M$.

\section{Remarks:}

1.Every projective module is pseudo projective module.

2.If every module has a quasi projective cover then it has a pseudo projective cover.

3 . If every module has a pseudo projective cover then it has a projective cover.

4. Every semi-simple projective module is pseudo projective module.

\section{Theorem :1.1}

Let $\mathrm{M}$ and $\mathrm{N}$ are two R-modules and assume $\mathrm{M}$ is $\mathrm{N}$-projective cover $\mathrm{P}$ via an onto homomorphism $\pi$ : $\mathrm{P} \longrightarrow \mathrm{M}$ then $\mathrm{M}$ is $\mathrm{N}$ projective iff for every homomorphism $\psi: \mathrm{P} \longrightarrow \mathrm{N}$, there exists a homomorphism $\phi: M \longrightarrow \mathrm{N}$ such that $\phi . \pi=\psi$. Equivalently $\psi(\operatorname{ker} \pi)=0$

Proof :

Only if direction :- Let $\psi: \mathrm{P} \longrightarrow \mathrm{N}$ is a homomorphism. We shall first show that $\psi(\operatorname{Ker} \pi)=0$. Let $\mathrm{T}=\psi(\operatorname{Ker} \pi)$ and Let $\pi_{\mathrm{T}}: \mathrm{N} \longrightarrow \frac{\mathrm{N}}{\mathrm{T}}$ be the natural projection. Then $\psi$ induces $\phi: M \longrightarrow \frac{\mathrm{N}}{\mathrm{T}}$ defined by $\phi(\mathrm{m})=\pi_{\mathrm{T}} \cdot \psi(\rho)$ where $M=\pi(\rho)$. Clearly $\phi \pi=\pi_{\mathrm{T}} \psi$. Since $M$ is N-projective, there exists a map $\beta: M \longrightarrow N$ such that $\phi=\pi_{\mathrm{T}} \cdot \beta$. Clearly $(\Psi-\beta \pi) P \subseteq T$. We claim that $\psi=\beta \pi$.

Let $x=\{\rho \in P \mid \psi(\rho)=\beta \pi(\rho)\}$. We show that $X=P$. Let $z \in P$.

Since $(\psi-\beta \pi)(x) \in T=\psi(\operatorname{Ker} \pi)$, there exists $k \in \operatorname{Ker} \pi$ such that $(\psi-\beta \pi)(\mathrm{x})=\psi(\mathrm{k})$. Therefore $\psi(\mathrm{z}-\mathrm{k})-\beta \pi(\mathrm{z}-\mathrm{k})=0$, since $\beta \pi(\mathrm{k})=0$. Thus $\mathrm{z}-\mathrm{k} \in \mathrm{X}$.

Therefore $\operatorname{Ker} \pi+X=P$ which implies $X=P$

Since Ker $\pi$ is small in $P$. Therefore $(\psi-\beta \pi) P=0$

In particular $(\psi-\beta \pi) \operatorname{Ker} \pi=0$, yielding $\psi(\operatorname{Ker} \pi)=0$. Equivalently, there exists $\psi^{\prime}: \mathrm{M} \longrightarrow \mathrm{N}$ such that $\psi_{\pi}^{\prime}=\psi$. 


\section{Conversely}

Let $\psi: \mathrm{M} \longrightarrow \frac{N}{k}$ is a homomorphism. Then by the projectivity of $\mathrm{P}$ there exists a homomorphism $\psi^{\prime}: \mathrm{P} \longrightarrow \mathrm{N}$ such that $\psi \pi=\pi_{\mathrm{k}} \psi^{\prime}$.

It follows easily that $\pi_{\mathrm{k}} \phi=\psi$ as desired. The above result is dual to a well-known characterization of relative injectivity.

\section{Definition: Pseudo weakly N-projective}

Let $\mathrm{M}$ and $\mathrm{N}$ are two R-modules and assume $\mathrm{M}$ has a projective cover $\quad \pi: \mathrm{P} \longrightarrow \mathrm{M}$. We say that $\mathrm{M}$ is pseudo weakly $\mathrm{N}$-projective if for every map $\psi: \mathrm{P} \longrightarrow \mathrm{N}$ there exists an epimorphism $\sigma: \mathrm{P} \longrightarrow \mathrm{M}$ and a homomorphism $\mathrm{g}: \mathrm{M} \longrightarrow \mathrm{N}$ such that $\psi=\mathrm{g} . \pi$.

\section{Theorem -1.2}

Let $\mathrm{M}$ and $\mathrm{N}$ are two R-modules and assume $\mathrm{M}$ has a projective cover $\quad \pi: \mathrm{P} \longrightarrow \mathrm{M}$. Then the following statements are equivalent :

1. $\quad \mathrm{M}$ is pseudo weakly $\mathrm{N}$-projective.

2. For every sub module $\mathrm{K} \subset \mathrm{N}, \mathrm{M}$ is pseudo weakly projective.

3. For every sub module $\mathrm{K} \subset \mathrm{N}, \mathrm{M}$ is pseudo weakly $\frac{\mathrm{N}}{\mathrm{K}}$-projective.

Proof :

(i) (1) Implies (2) and (3) Assume $\mathrm{M}$ is pseudo weakly $\mathrm{N}$-projective and let $\mathrm{K}$ is a sub-module of $\mathrm{N}$ and $\psi: \mathrm{P} \longrightarrow \mathrm{K}$ is a homomorphism. Then $\psi=\mathrm{i}_{\pi} . \quad \psi: \mathrm{P} \longrightarrow \mathrm{N}$ may be expressed as a composition $\psi=\mathrm{g} \sigma$ for some homomorphism $\mathrm{g}: \mathrm{M} \longrightarrow \mathrm{N}$ and epimorphism $\sigma: \mathrm{P} \longrightarrow \mathrm{M}$. Since $\sigma$ is onto, the range of $\sigma$ equals the range of $\mathrm{g}$ and so it is contained in $\mathrm{K}$. Thus we may define $\mathrm{g}: \mathrm{M} \longrightarrow \mathrm{K}$ via $\psi(\mathrm{m})=\mathrm{g}(\mathrm{m})$ and then $\psi=\mathrm{g} \sigma$, proving that $\mathrm{M}$ is pseudo weakly $\mathrm{K}$-projective as claimed. Assume once again that $\mathrm{M}$ is pseudo weakly $\mathrm{N}$-projective and let $\mathrm{f}: \mathrm{P} \longrightarrow \mathrm{N} / \mathrm{K}$ is a homomorphism. Since $\mathrm{P}$ is projective, there exists a map $\overline{\mathrm{f}}: P \longrightarrow N$ such that $\mathrm{f}=\pi_{x} . \overline{\mathrm{f}}$. The weakly N-projective of $\mathrm{M}$ yields an epimorphism $\sigma: \mathrm{P} \longrightarrow \mathrm{M}$ and a homomorphism $\mathrm{h}: \mathrm{M} \longrightarrow \mathrm{N}$ such that $\overline{\mathrm{f}}=\mathrm{h} . \sigma$. Let $\pi_{\underline{x}} \cdot \mathrm{h}=\mathrm{f}_{1}$ then $\mathrm{f}_{1} \sigma=\pi_{\mathrm{k}} \cdot \mathrm{h} \cdot \sigma=\pi \overline{\mathrm{f}}=\mathrm{f}$, proving that $\mathrm{M}$ is indeed pseudo weakly $\frac{N}{K}$ projective.

(ii) (2) or (3) implies (1) is trivially.

\section{Remarks :}

Let $\mathrm{M}$ and $\mathrm{N}$ are two R-modules and assume $\mathrm{M}$ has a projective cover $\quad \pi: \mathrm{P} \longrightarrow \mathrm{M}$. Then $\mathrm{M}$ is pseudo weakly N-projective if and only if for every sub-module $\mathrm{K} \subset \mathrm{N}$ and for every epimorphism $\psi: \mathrm{P} \longrightarrow \mathrm{K}$ there exist epimorphism $\sigma: \mathrm{P} \longrightarrow \mathrm{M}$ and $\mathrm{g}: \mathrm{M} \longrightarrow \mathrm{N}$ such that $\psi=\mathrm{g} . \sigma$.

\section{Theorem:1.3}

Let $\mathrm{M}$ and $\mathrm{N}$ are two R-modules and assume $\mathrm{M}$ has a projective cover $\pi: \mathrm{P} \longrightarrow \mathrm{M}$. Then $\mathrm{M}$ is pseudo weakly N-projective iff for every map $\psi: \mathrm{P} \longrightarrow \mathrm{N}$ there exist a sub module $\mathrm{X} \subset \operatorname{Ker} \psi$ such that $\frac{P}{X} \cong M$.

\section{Proof :}

Necessary condition :

Let $\psi: \mathrm{P} \longrightarrow \mathrm{N}$ is a homomorphism. Assume $\mathrm{M}$ is pseudo weakly $\mathrm{N}$-projective and let the homomorphism $\mathrm{g}: \mathrm{M} \longrightarrow \mathrm{N}$ and the epimorphism $\sigma: \mathrm{P} \longrightarrow \mathrm{M}$ be as in the definition of weakly relative projectivity. Since $\psi=$ g. $\sigma$, Ker $\sigma \subset \operatorname{Ker} \psi$. Also $\frac{P}{\operatorname{Ker} \sigma} \cong M$ Thus the implication is proven by choosing $\mathrm{X}=\operatorname{Ker} \sigma$. 


\section{Conversely :}

If $\mathrm{X} \subset \mathrm{P}$ satisfies the condition in the statement of the theorem, then the isomorphism $\frac{P}{X} \cong M$, composed with the natural projection $\pi_{\mathrm{k}}: \mathrm{P} \longrightarrow \mathrm{P} / \mathrm{X}$ is an epimorphism $\sigma: \mathrm{P} \longrightarrow \mathrm{M}$ satisfying that Ker $\sigma=X \subset \operatorname{Ker} \psi$. It follows that the map $g: M \longrightarrow N$ given by $g(m)=\psi(\rho)$ whenever $\sigma(\rho)=M$ is well defined and satisfies $\psi=$ g. $\sigma$

\section{Theorem :1.4}

Let $\mathrm{M}$ and $\mathrm{N}$ are two R-modules and assumed $\mathrm{M}$ is supplemented and has a projective cover

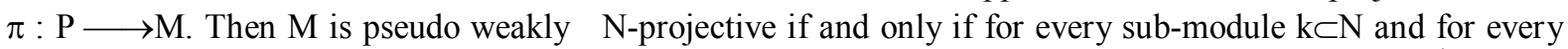
epimorphism $\psi: \mathrm{P} \longrightarrow \mathrm{K}$ there exists an epimorphism $\mathrm{g}: \mathrm{M} \longrightarrow \mathrm{K}$ such that for every supplement $\mathrm{L}^{\prime}$ of Kerg in $\mathrm{M}$ there exists a sub-module $\mathrm{L} \subset \mathrm{P}$ such that $\frac{P}{L} \cong \frac{M}{L^{\prime}}$ and $\mathrm{L}+\mathrm{Ker} \psi=\mathrm{P}$.

Proof :

\section{Necessary Condition :}

Assume $\mathrm{M}$ is pseudo weakly $\mathrm{N}$-projective and Let $\psi: \mathrm{P} \longrightarrow \mathrm{K}$ is an epimorphism onto a sub-module $\mathrm{K} \subset \mathrm{N}$. Then there exists epimorphism $\sigma: \mathrm{P} \longrightarrow \mathrm{M}$ and $\mathrm{g}: \mathrm{M} \longrightarrow \mathrm{K}$ such that $\psi=\mathrm{g} \sigma$. Let L' is a supplement of Kerg in $M$ and $L=\sigma^{-1}\left(L^{\prime}\right)$. For an arbitrary $p \in P, \sigma(p)$ may be written as $\sigma(P)=1^{\prime}+k^{\prime}$, with $l^{\prime} \in L^{\prime}$ and $k^{\prime} \in \operatorname{Kerg}$. It follows then that $\psi(\mathrm{p})=\mathrm{g} \sigma(\mathrm{p})=\mathrm{g}\left(\mathrm{l}^{\prime}\right)+\mathrm{g}\left(\mathrm{k}^{\prime}\right)=\mathrm{g}\left(\mathrm{l}^{\prime}\right)$.

Choose $\mathrm{p}_{1} \in \sigma^{-1}\left(\mathrm{l}^{\prime}\right) \subset \mathrm{L}$. Then $\sigma\left(\mathrm{p}_{1}\right)=\mathrm{l}^{\prime}$. On the other hand,

$\psi\left(\mathrm{p}_{1}\right)=\mathrm{g} \sigma\left(\mathrm{p}_{1}\right)=\mathrm{g}^{\prime}\left(\mathrm{l}^{\prime}\right)=\psi(\mathrm{p})$. So P-P $\mathrm{P}_{1} \in \operatorname{Ker} \psi$ and so $\mathrm{L}+\operatorname{Ker} \psi=\mathrm{P}$. The fact that $\frac{P}{L} \cong \frac{M}{L^{\prime}}$ follows, since $\mathrm{L}$ is the kernel of the onto map $\pi_{\mathrm{L} .} \sigma: \mathrm{P} \longrightarrow \frac{M}{L^{\prime}}$.

\section{Sufficient Condition :}

Let us assume that for every sub-module $\mathrm{K} \subset \mathrm{N}$ and for every epimorphism $\psi: \mathrm{P} \longrightarrow \mathrm{K}$ there exist an

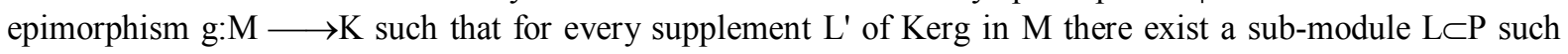
that $\frac{P}{L} \cong \frac{M}{L^{\prime}}$ and $\mathrm{L}+\mathrm{Kerg}=\mathrm{P}$. Let $\psi: \mathrm{P} \longrightarrow \mathrm{K}$ is an epimorphism and $\mathrm{g}: \mathrm{M} \longrightarrow \mathrm{K}$ be the corresponding epimorphism. All we need is to produce another epimorphism $\sigma: \mathrm{P} \longrightarrow \mathrm{M}$ such that $\psi=\mathrm{g} \sigma$. Let L' is a supplement for Kerg and Let $\mathrm{L}$ be the corresponding sub-module of P. Let $\theta: \frac{P}{L} \longrightarrow \frac{M}{L^{\prime}}$ is an isomorphism. By Chinese remainder theorem that the map $\mathrm{M}+\operatorname{Kerg} \cap \mathrm{L}^{\prime} \longrightarrow\left(\mathrm{M}+\mathrm{Kerg}, \mathrm{M}+\mathrm{L}^{\prime}\right)$ is an isomorphism between $\frac{M}{\left(\operatorname{Ker} g \cap L^{\prime}\right)}$ and $\frac{M}{\left(\operatorname{Ker} g \times \frac{M}{L^{\prime}}\right)}$.

Also $\frac{M}{\operatorname{Ker} g} \cong K$ via $\mathrm{M}+\operatorname{Ker} \mathrm{g}=\mathrm{g}(\mathrm{m})$

$\mathrm{g}=\theta \pi_{\mathrm{L}}: \mathrm{P} \longrightarrow \frac{M}{L^{\prime}}$. Since Ker $\psi+\mathrm{L}=\mathrm{P}$, the map $\alpha: \mathrm{P} \longrightarrow K \times \frac{M}{L^{\prime}}$ given by $\alpha(\mathrm{p})=(\psi(p), g(p))$ is onto.

The induced epimorphism $\alpha^{1}=\beta^{-1} \alpha: P \longrightarrow \frac{M}{\left(\operatorname{Kerg} \cap L^{\prime}\right)}$ may then the lifted to a map $\sigma: \mathrm{P} \longrightarrow \mathrm{M}$. Since Kerg $\cap \mathrm{L}^{\prime} \leq \mathrm{M}$. $\sigma$ is indeed an epimorphism. It only remains to show that g. $\sigma=\psi$. Let us refer for the rest of this proof to $\pi_{\mathrm{Kerg} \cap L^{\prime}}$ simply as $\pi$. We know that $\pi \sigma=\sigma^{\prime}=\beta^{\prime} \alpha$ hence $\beta \pi \sigma=\alpha$. Let $p \in P$ be 
arbitrary. Then $\beta\left(\alpha(P)+\operatorname{Ker} g \cap L^{\prime}\right)=\alpha(p)=(\psi(p), g(p))$. On the other hand $\beta\left(\sigma(P)+\operatorname{Kerg} \cap L^{\prime}\right)=(g(\sigma(p))$, $\left.\sigma(p)+L^{\prime}\right)$. Comparing the first component in both expression yields the desired equality. Thus $M$ is pseudo weakly N-projective.

\section{References}

[1]. Dinguuo Wang and Jinqui Li: Characterization of rings using weakly projective module. Vietnam journal of mathematics.vol.25.(1998)

[2]. Quasi weakly projective and quasi weakly injective module: M.R. Aloney S. Jat. Ultra scientist journal of physical sciences.(2011)

[3]. A.K.Tiwary and B.M.Pandeya: Pseudo projective and pseudo injective modules. B.H.U. Vol.9 No.9(1978)

[4]. S. K. Jain, Lopez - Permouth, K. Oshiro and M. A. Saleh : "Weakly Projective and Weakly Injective Modules." Can. J. Math. Vol 46(5) (1994) 971-981.

[5]. S. R. Lopez - Permouth : Ring Characterized by their Weakly Injective Modules, Glasgow Math. J. 34 (1992) $349-353$.

[6]. D.Wang: Characterization of rings using weakly projective module I. Vietnam journal of mathematics.vol.25.(1997) 91-98. 